\title{
Presión tributaria, gasto público e inversión privada en la Comunidad Andina de Naciones
}

\author{
Tax burden, public expenditure, and private \\ investment in the Andean Community
}

Luis Felipe Brito Gaona Universidad Técnica de Machala (Machala - Ecuador) lbrito@utmachala.edu.ec

Emma M. Iglesias Universidad de La Coruña (España) emma.iglesias@udc.es

Revista Cumbres Vol.3 №2

Versión impresa ISSN 1390-9541

Versión electrónica ISSN 1390-3365

http://investigacion.utmachala.edu.ec/revistas/index.php/Cumbres 


\section{RESUMEN}

Este artículo busca evidencia empírica acerca de si un aumento de los impuestos, del gasto público y en general, un estado muy intervencionista estimula o desestimula la inversión privada en los países de la Comunidad Andina de Naciones (CAN). Usando los mismos determinantes de la inversión privada que Caballero-Urdiales y López-Gallardo (2012), realizamos el análisis de los 4 países que conforman la CAN. Los resultados a partir de nuestras elasticidades estimadas muestran evidencia consistente con tres hipótesis: (1) que a corto plazo, la presión tributaria (impuestos sobre la renta y consumo) tiene efectos significativos en la inversión privada mientras que a largo plazo sólo el gasto público y el tipo de cambio son significativas; (2) que la inversión pública tiene un efecto de crowding out con la inversión privada tanto a corto como a largo plazo; (3) y que para estimular la inversión privada, se prefiere que el gobierno sea poco intervencionista tanto a corto como a largo plazo. Para este grupo de países seleccionado se utilizó una especificación econométrica basada en un modelo de panel dinámico para el periodo comprendido entre 1990 y 2012, en donde la inversión privada es la variable dependiente del producto interno bruto o ingreso, del gasto público, de la recaudación tributaria por concepto de impuesto sobre la renta, del impuesto al valor agregado, y el índice de tipo de cambio real.

Palabras clave: Inversión, PIB, Gasto público, ISR, IVA.

\section{ABSTRACT}

This work seeks empirical evidence on whether an increase in taxes, public spending and, in general, a highly interventionist state stimulates or discourages private investment in the countries of the Andean Community of Nations (CAN). Using the same determinants of private investment as Caballero-Urdiales and López-Gallardo (2012), we performed the analysis of the 4 countries that make up the CAN. The results from our estimated elasticities show evidence that is consistent with three hypotheses: (1) in the short term, tax burden (taxes on income and consumption) has a significant effect on private investment, whereas in the long run only public expenditure and the exchange rate are significant; (2) public investment has a crowding out effect on private investment both in short and long terms; and (3) to motivate private investment, it is preferred that the government has little intervention in both short or long terms. For this selected group of countries, an econometric specification based on a dynamic panel model was used for the period between 1990 and 2012, where private investment is the dependent variable of gross domestic product or income, public expenditure, revenue income tax, value added tax, and the real exchange rate index.

Keywords: Private investment, GDP, public expenditure, RIT, VAT. 


\section{INTRODUCCIÓN}

Este artículo explora opiniones acerca de la inversión privada en los países que conforman la Comunidad Andina de Naciones (CAN). Estas opiniones han intentado explicar cómo la inversión privada aporta al crecimiento económico de corto y largo plazo, a partir de situar los determinantes de la inversión privada como generador de riqueza y no a los de ahorro, como tradicionalmente se asume, en las cuales nos proponemos explicar tres hipótesis: (1) a corto plazo, la presión tributaria (impuestos sobre la renta y consumo) tiene efectos significativos en la inversión privada mientras que a largo plazo sólo el gasto público y el tipo de cambio son significativas; (2) la inversión pública tiene un efecto de crowding out con la inversión privada tanto a corto como a largo plazo; (3) para estimular la inversión privada, se prefiere que el gobierno sea poco intervencionista tanto a corto como a largo plazo.

En relación a la primera de las hipótesis, Harberger (1964) había postulado que la teoría predice que cambios en la presión tributaria afectan significativamente a la inversión privada en el largo plazo. Mendoza et al. (1997) muestran evidencia teórica, en la que usando una panel de países no latinoamericanos en el periodo 1966-1990, tiene resultados a favor de dicha hipótesis. En relación al signo de dicha relación, Mendoza et al. (1997), demuestran que en conjunto, un aumento de la presión tributaria reduce la inversión privada, y en especial, un aumento de los impuestos al consumo (impuesto al valor agregado) aumentan de manera significativa la inversión privada. La razón teórica que dan para dicha relación positiva es que si se incrementan los impuestos al consumo, se consume menos, y se dedica más parte del output total a la inversión privada dado que el consumo es más caro (este resultado depende de si el capital y el consumo son sustitutivos perfectos o no -ver Pecorino (1993) y Mendoza et al. (1997)-. Caballero-Urdiales y López-Gallardo (2012) analizan un panel de cinco países latinoamericanos (Brasil, Chile, Colombia, México y Uruguay) en el periodo 1990-2008 y también encuentran evidencia a favor de que un aumento del impuesto sobre la renta y del impuesto sobre el valor añadido disminuyen de manera significativa la inversión privada, tanto a corto como a largo plazo. Además, encuentran evidencia a favor de que el impuesto sobre el valor añadido tiene una relación negativa con la inversión privada. Sin embargo, también hay autores como Pecho-Trigueros y Peragón-Lorenzo (2013) que afirman que la carga tributaria no tiene un efecto directo sobre la inversión privada o que la inversión privada se ve afectada por la carga tributaria cuando ésta supera cierto porcentaje.

En relación a la segunda de las hipótesis de cómo afecta la inversión pública a la inversión privada, Gutiérrez (1996) muestra evidencia para el caso de Chile de que un aumento de la inversión pública genera un desplazamiento de la inversión privada (crowding out). Mendoza et al. (1997) no encuentra un efecto significativo entre las compras del gobierno y la inversión privada, mientras que por el contrario, Caballero-Urdiales y López-Gallardo (2012) concluyen que para los cinco países latinoamericanos, el gasto público tiene un efecto positivo sobre la inversión privada (tanto a corto como a largo pla- 
zo), es decir, que se da un efecto atracción (crowding in), con lo cual se rechaza el efecto expulsión (crowding out) que postula la teoría convencional. Brito e Iglesias (2017a) concluyen que para los países latinoamericanos, el gasto público tiene un efecto negativo sobre la inversión privada (tanto a corto como a largo plazo), es decir, que se da un efecto expulsión. Brito e Iglesias (2017b) concluyen que para los países de la Unión Europea, el gasto público tiene un efecto positivo sobre la inversión privada (tanto a corto como a largo plazo), es decir, que se da un efecto atracción, con lo cual se rechaza el efecto expulsión (crowding out) que postula la teoría convencional.

Finalmente, la tercera de las hipótesis que pretendemos contrastar, consiste en analizar el efecto neto del papel del estado vía impuestos y el efecto de la inversión pública en la inversión privada. Mendoza et al. (1997) encuentran evidencia a favor de un efecto negativo neto para el papel del estado en la inversión privada, por el contrario, Caballero-Urdiales y López-Gallardo (2012).

Esta importante diferencia de opiniones abre un espacio para la discusión y para ello se ha recolectado evidencia empírica y teórica sobre varios contextos ajenos al ecuatoriano y el impacto que han tenido los impuestos en estos, del mismo modo, se ha mostrado de manera estadística el impacto de cada una de las variables estudiadas en la búsqueda de estimular la inversión privada. Caballero y López (2012) manifiesta en su artículo que hay que dar a los inversionistas las mayores facilidades pero no con tasas impositivas muy pequeñas, sino asegurando un mercado en expansión, infraestructura moderna, créditos baratos entre otros estímulos que solo un Estado fuerte, con recursos y bien organizado puede brindar.

El objetivo principal de este trabajo ha sido mostrar el impacto que han tenido el gasto público, el producto interno bruto y los impuestos sobre la inversión privada en estos países durante los últimos años y cuáles de estas variables han afectado a la inversión privada, y mostrar que la estrategia de aumentar la participación del Estado en la economía, ha sido equivocada. Especificamente queremos probar la hipótesis de que el gasto gubernamental y el impuesto a la renta desestimulan la inversión privada, siempre que estos no se redistribuyan correctamente para crear nueva demanda.

Según Jiménez (2015), la evolución de la estructura tributaria en los países de América Latina, ha resultado, en la mayoría de los casos, en una relación muy desigual entre la imposición directa e indirecta. En este sentido, la política tributaria no solo ha fortalecido la imposición general al consumo mediante el fortalecimiento del IVA, además, la imposición a la renta se orientó a gravar básicamente los ingresos de las personas jurídicas y, en menor medida, las rentas obtenidas por las personas naturales. Todos estos resultados contrastan drásticamente con los que muestran los países desarrollados de la Organización de Cooperación y Desarrollo Económicos (OCDE), en donde la presión tributaria media, que es considerablemente mayor (un 34,3\% en el período 2001-2011), se compone en un $42 \%$ de impuestos directos y un 31,6\% de imposición indirecta.

Según Carrasco (2012), ex director del Servicio de Rentas Internas del Ecuador (SRI) la manera más eficiente de redistribución de riqueza es me- 
Tabla 1. CAN: Presión tributaria en porcentaje del PIB

\begin{tabular}{|l|l|l|l|l|l|l|l|l|}
\hline & $\mathbf{1 9 9 0}$ & $\mathbf{2 0 0 0}$ & $\mathbf{2 0 0 7}$ & $\mathbf{2 0 0 8}$ & $\mathbf{2 0 0 9}$ & $\mathbf{2 0 1 0}$ & $\mathbf{2 0 1 1}$ & $\mathbf{2 0 1 2}$ \\
\hline Bolivia & $\mathbf{7 , 2}$ & $\mathbf{1 4 , 7}$ & 22,6 & 20,5 & 22,7 & 20,7 & 24,2 & 26,0 \\
\hline Colombia & 9,0 & 14,6 & 19,1 & 18,8 & 18,6 & 18,0 & 18,8 & 19,6 \\
\hline Ecuador & 7,1 & 10,1 & 12,8 & 14,0 & 14,9 & 16,8 & 17,9 & 20,2 \\
\hline Perú & 11,8 & 13,9 & 17,8 & 18,2 & 16,3 & 17,4 & 17,8 & 18,1 \\
\hline América Latina & 13,6 & 16,4 & 19,5 & 19,5 & 18,9 & 19,3 & 20,1 & 20,7 \\
\hline
\end{tabular}

Fuente: OCDE, CEPAL, CIAT (2014)

diante leyes tributarias, de tal manera que se puede financiar el gasto social con estos recursos. Pero se convierte en un problema cuando es un desincentivo a la inversión, cuando reduce las actividades productivas y por consecuencia genera una reducción en la generación de empleo.

Según la CEPAL, los ingresos fiscales de la región siguen siendo bajos, como parte del PIB nacional. En 2013 los ingresos tributarios llegaron al $21,3 \%$, con un aumento mínimo respecto al año anterior de $20,7 \%$. Si bien el crecimiento paulatino de la recaudación tributaria ha dotado a los gobiernos de una mayor capacidad para incrementar el gasto en programas sociales e infraestructuras en las últimas décadas, la cifra se encuentra todavía 14 puntos porcentuales por debajo de la media de los países de la OCDE, del $34,1 \%$ pero se reconoce el incremento experimentado durante el período 1990-2012: la presión tributaria creció en siete puntos porcentuales en 23 años, de 13,6\% a 20,7\%, como se puede apreciar en la Tabla 1.

Como observamos en la Tabla 2 y 3, la recaudación por concepto de impuestos al consumo (impuestos sobre bienes y servicios), en el promedio de los países latinoamericanos, representó el 10,5\% del PIB durante el 2012, mientras que los impuestos al ingreso (impuestos sobre las rentas y ganancias de capital), ascendieron a 5,2\% en ese mismo año, aumento sostenido según se observa en las tablas.

Tabla 2. CAN: Impuesto sobre la renta y utilidades en porcentaje del PIB

\begin{tabular}{|l|l|l|l|l|l|l|l|l|}
\hline & $\mathbf{1 9 9 0}$ & $\mathbf{2 0 0 0}$ & $\mathbf{2 0 0 7}$ & $\mathbf{2 0 0 8}$ & $\mathbf{2 0 0 9}$ & $\mathbf{2 0 1 0}$ & $\mathbf{2 0 1 1}$ & $\mathbf{2 0 1 2}$ \\
\hline Bolivia & 0,8 & $\mathbf{1 , 9}$ & 3,0 & 3,8 & 3,9 & 3,9 & 3,8 & 4,0 \\
\hline Colombia & 2,7 & 3,4 & 5,6 & 5,1 & 5,6 & 4,8 & 5,4 & 6,6 \\
\hline Ecuador & 0,9 & 1,6 & 2,5 & 3,8 & 4,0 & 3,5 & 3,9 & 3,9 \\
\hline Perú & 0,7 & 2,8 & 7,3 & 7,1 & 5,9 & 6,5 & 7,5 & 7,7 \\
\hline América Latina & 3,0 & 3,2 & 4,9 & 4,9 & 4,7 & 4,6 & 4,9 & 5,2
\end{tabular}

Fuente: OCDE, CEPAL, CIAT (2014)

De acuerdo a Arévalo (2014), menciona que dentro del contexto económico, político y social de América Latina de los últimos tres lustros y especialmente en países como Venezuela, Ecuador, Bolivia, Nicaragua y Argentina, se ha dado paso a nuevas posturas ideológicas y políticas, cuyo propósito fue el de experimentar con nuevos modelos de desarrollo económico con la pretensión de solucionar urgentes problemas de carácter estructural rela- 


\begin{tabular}{l|l|l|l|l|l|l|l|l|} 
Tabla 3. CAN: Impuesto sobre bienes y servicios en porcentaje del PIB \\
\hline & $\mathbf{1 9 9 0}$ & $\mathbf{2 0 0 0}$ & $\mathbf{2 0 0 7}$ & $\mathbf{2 0 0 8}$ & $\mathbf{2 0 0 9}$ & $\mathbf{2 0 1 0}$ & $\mathbf{2 0 1 1}$ & $\mathbf{2 0 1 2}$ \\
\hline Bolivia & 5,1 & 10,0 & 16,5 & 16,4 & 14,1 & 14,5 & 16,5 & 17,0 \\
\hline Colombia & 4,8 & 6,4 & 8,1 & 8,2 & 7,5 & 7,7 & 7,8 & 7,6 \\
\hline Ecuador & 4,4 & 7,3 & 6,8 & 6,8 & 7,2 & 9,1 & 8,7 & 10,5 \\
\hline Perú & 7,8 & 8,5 & 7,8 & 8,2 & 7,4 & 8,0 & 7,8 & 7,7 \\
\hline América Latina 2 & 7,1 & 9,2 & 10,2 & 10,3 & 9,5 & 10,0 & 10,3 & 10,5 \\
\hline
\end{tabular}

Fuente: OCDE, CEPAL, CIAT (2014)

cionados con el crecimiento, el desempleo, la lucha contra la pobreza y la inequidad del ingreso.

Los datos evaluados en los párrafos anteriores permiten destacar dos postulados importantes; el primero relativo a la importancia de la inversión pública como motor del crecimiento económico en los últimos años; el segundo sobre la evolución de la inversión privada y el debate en torno a las políticas públicas necesarias para su promoción.

Caballero y López (2012) indican que una evolución macroeconómica desfavorable se explica, en muy alta medida, por el lento crecimiento de la acumulación productiva y, en concreto, por el lento ritmo de expansión de la inversión y esto lleva a formularse la pregunta de qué estrategia debería seguirse para estimular la inversión.

Pérez (2011), en su análisis del modelo Kaleckiano menciona que un aumento de la inversión puede lograrse por medio de la intervención del gobierno; que con reglas claras de comportamiento el nivel de intervención necesaria por parte del Estado puede ser financiado por medio de impuestos sobre las ganancias, sin que esto lleve a un desplazamiento neto de recursos (crowding out), situación que se rechaza dados los resultados del presente trabajo.

\section{MATERIALES Y MÉTODOS}

En esta sección se utilizó una especificación econométrica basada en un modelo de panel dinámico para el periodo comprendido entre 1990 y 2012 en los países que conforman la Comunidad Andina de Naciones (CAN) Bolivia, Colombia, Ecuador y Perú, en donde la inversión privada (ip), es la variable dependiente del producto interno bruto o ingreso (pib), del gasto público (g), de la recaudación tributaria por concepto de impuesto sobre la renta (isr), de la recaudación tributaria por concepto del impuesto al valor agregado (iva), y el índice de tipo de cambio real (tc) y de esta manera medir el impacto de las variables fiscales sobre la inversión privada en dicho periodo.

Se utilizaron serie de datos anuales de longitud 23 para un panel de cuatro países desde 1990 hasta 2012. A precios constantes en dólares. Estas series se obtuvieron de la Comisión Económica para América Latina y el Caribe (CEPAL), en la sección de cuentas nacionales y estadísticas de finanzas

\section{Cumbres}


públicas. Por lo tanto: $\mathrm{i}=1, \ldots, 4$ (países seleccionados) y $\mathrm{t}=1, \ldots . ., 23$ (años desde 1990 hasta 2012).

La selección de países integrantes de la CAN se realizó de acuerdo a varios criterios vinculados al comercio entre ellos, libre movilidad, características estructurales similares y distintos modelos de tributación y moneda lo que reduce el riesgo de introducir en el análisis problemas de heterogeneidad estructural. Para el tratamiento de datos, se utilizó el programa estadístico Stata 14.

Se incluye el PIB y el tipo de cambio real para evitar el sesgo de variables no consideradas en la especificación de la función de inversión, y para tomar en cuenta, en alguna medida, el carácter abierto de las economías de los países tomados en consideración.

Los resultados de corto plazo del modelo dinámico se pueden visualizar en la Tabla 4 y las variables consideradas son significativas con un valor de la probabilidad del estadístico (t-prob) inferior a 0,05.

El panel se encuentra balanceado y en la serie de datos se encuentran todos los países para todos los años y todas las variables considerados y se utilizó el método de estimación entre efectos fijos y efectos aleatorios, que tienen mejores propiedades que el método de momentos documentadas en el libro de Wooldridge (2010).

A las variables estudiadas se les realizaron transformaciones logarítmicas, se hicieron pruebas de efectos fijos y efectos aleatorios, brindando como resultado la aplicación de efectos fijos según el test de Hausman (1978) para contrastar la hipótesis nula, en la que nos indica que el modelo preferido es el de efectos fijos en lugar de efectos aleatorios. Posteriormente se le realizó el test de Wooldridge (2002), la misma que indicó que no existe autocorrelación. La prueba AR(1), mostrada en la tabla 4 como m1, demuestra que no existe correlación de orden 1 entre los términos de error al 5\%.

Como se observa en la Tabla 4, el modelo pasa todas las pruebas. La prueba del contraste de Wald (joint) indica que todas las variables son significativas conjuntamente.

\section{RESULTADOS Y DISCUSIÓN}

\section{Análisis econométrico a corto plazo}

Se obtienen los siguientes resultados del panel:

Se observa un efecto acelerador en la medida que el alza del ingreso genera un incremento de la inversión privada. Los resultados indican que el aumento del $1 \%$ del PIB de un periodo se traduce en un aumento de 1,14\% inicial de la inversión privada sin rezago, pero se da una disminución de 0,38\% de la inversión privada, como consecuencia del alza del PIB con un rezago de siete periodos, por lo que el balance es positivo.

El gasto público tiene un efecto negativo sobre la inversión privada, es decir se da un efecto expulsión, con lo cual se acepta la hipótesis de dicho efecto (crowding out), que postula la teoría convencional. Los resultados 
Tabla 4. Elasticidades de corto plazo para la ecuación de inversión privada

Variable dependiente: Inversión privada

Método de estimación: Efectos fijos

Periodo: 1990- 2012

Países considerados: Bolivia, Colombia, Ecuador, Perú

\begin{tabular}{|l|c|l|}
\hline VARIABLES INDEPENDIENTES & & T-PROB \\
\hline Inversión privada (-1) & 0,5750 & 0,000 \\
\hline Inversión privada (-2) & 0,0312 & 0,279 \\
\hline Ingreso & 1,1418 & 0,000 \\
\hline Ingreso (-7) & $-0,3750$ & 0,001 \\
\hline Gasto público (-1) & $-0,6761$ & 0,000 \\
\hline Gasto público (-7) & 0,2568 & 0,001 \\
\hline Impuesto sobre la renta (-3) & $-0,0231$ & 0,019 \\
\hline Impuesto al valor agregado & 0,1679 & 0,000 \\
\hline Impuesto al valor agregado (-7) & $-0,0641$ & 0,002 \\
\hline Índice del tipo de cambio real (-7) & $-0,2715$ & 0,006 \\
\hline Wald (joint) & 167,53 & \\
\hline & {$[0,000]$} & \\
\hline m1 & 9,926 & \\
\hline & {$[0,0512]$} & \\
\hline Número de observaciones & 85 & \\
\hline Notas: & & \\
\hline i) las variables se usaron en transformación logarítmica; & & \\
\hline ii) para los contrastes se reportan los valores en corchetes. & & \\
\hline
\end{tabular}

muestran que el aumento de un punto porcentual del gasto público se traduce en una disminución de la inversión privada de 0,68\% con rezago de un periodo y un aumento de $0,26 \%$ de la inversión privada con un rezago de siete periodos.

El impuesto sobre la renta desestimula la inversión privada, de acuerdo con los resultados del modelo, con un alza de 1\% en la recaudación tributaria por este concepto, la inversión privada de reduce 0,02\% con un rezago de tres periodos.

El impuesto al valor agregado según el modelo, con un alza de $1 \%$ en la recaudación, la inversión privada se incrementa $0,17 \%$ en el mismo período. Pero disminuye $0,06 \%$ la inversión privada con un rezago de siete periodos. Con esto se rechaza la hipótesis de la superioridad del impuesto al valor agregado sobre el impuesto a la renta, al suponerse que es neutral respecto a las decisiones de inversión privada. Mendoza et al. (1997) demuestran que los impuestos al capital y los impuestos al trabajo (a la renta) pueden tener una relación negativa con la inversión privada mientras que los impuestos al consumo (impuesto al valor agregado), tienen una relación positiva. La razón teórica que dan es que si se incrementan los impuestos al consumo, se consume menos, y se dedica más parte del output total a la inversión privada dado que el consumo es más caro.

Los resultados indican que el gasto público y los impuestos en la inversión privada, reflejan un balance fiscal negativo en el grupo de países analizados. 
Como se aprecia en los párrafos anteriores, el efecto estimulante del impuesto al valor agregado sobre la inversión privada, supera al efecto del gasto público y del impuesto sobre la renta. Lo cual implica que si se incrementara la recaudación por impuesto a la renta para incrementar el gasto público, se tendría una disminución de la inversión privada. Si se incrementara el impuesto al valor agregado para incrementar el gasto público, en cambio afectaría positivamente a la inversión privada, pero el efecto final sería negativo.

Por último, el tipo de cambio real ejerce un efecto negativo sobre la inversión privada, resultado consistente obtenido por López, Spanos y Sánchez (2011), y en sentido contrario al obtenido por Ibarra (2008, 2009). En concreto, se observa que un incremento de $1 \%$ del índice de tipo de cambio real, con un rezago de cuatro periodos produce un aumento de la inversión privada de $0,27 \%$.

\section{Análisis econométrico a largo plazo}

En los resultados del panel se pudo encontrar una relación de largo plazo como lo realiza Caballero y López (2012) dado que se logró encontrar resultados significativos en el ingreso, en el gasto público y en el tipo de cambio. Los resultados de largo plazo revelan el efecto total que producen los cambios de las variables independientes sobre la inversión privada, es decir, se compara una nueva situación de equilibrio respecto de la que existía antes de que se produjeran los incrementos de las variables.

Se observa en la tabla 5, que el PIB tiene un efecto acelerador dado que el coeficiente de elasticidad ingreso de la inversión privada es alto, coincidiendo con Caballero y López (2012). El gasto público tiene un efecto negativo sobre la inversión privada, lo cual se puede concluir que es independiente de la forma como se financie. El índice de tipo de cambio real desestimula la inversión privada, lo cual coincide con los resultados obtenidos en otros países, utilizando similares metodologías como Caballero y López (2012) para el caso mexicano.

Tabla 5. Elasticidades de largo plazo para la ecuación de inversión privada

Variable dependiente: Inversión privada (lip)

Periodo: 1990- 2012

Países considerados: Bolivia, Colombia, Ecuador, Perú

\begin{tabular}{l|l|l}
\hline VARIABLES INDEPENDIENTES & & \\
\hline Ingreso (lpib) & $1,8180(0,1301)$ & {$[13,98]$} \\
\hline Gasto público $(\operatorname{lgp})$ & $-0,4359(0,1105)$ & {$[-3,95]$} \\
\hline $\begin{array}{l}\text { Índice del tipo de cambio real } \\
\text { (ltc) }\end{array}$ & $-0,6707(0,1293)$ & {$[-5,19]$} \\
\hline $\begin{array}{l}\text { Notas: se reportan el coeficiente de largo plazo, el error estándar de largo plazo en paréntesis y el } \\
\text { valor estadístico t en corchetes. }\end{array}$
\end{tabular}




\section{CONCLUSIONES}

Los resultados del análisis realizados en este artículo para los países de la CAN muestran evidencia a favor de las siguientes tres hipótesis: Primero, que la presión tributaria tiene efectos significativos en la inversión privada en el corto plazo. Segundo, que la inversión pública tiene un claro efecto crowding out con la inversión privada tanto en el corto como en el largo plazo. Esta es la misma hipótesis que la apoyada por Gutiérrez (1996) para Chile y contraria a la apoyada en Caballero-Urdiales y López-Gallardo (2012). Tercero, que para estimular la inversión privada, tanto en el corto como en el largo plazo, se prefiere que el gobierno sea poco intervencionista, hipótesis contraria a la apoyada en Caballero-Urdiales y López-Gallardo (2012).

Estos resultados implican primero que el gasto público es incapaz de reactivar la inversión privada, sobre todo cuando es financiado con recursos tributarios. De esta manera, mostramos evidencia a favor de que la posición durante las últimas décadas que han sostenido algunos gobiernos latinoamericanos, en el sentido de aumentar la participación estatal en la actividad económica del país y privilegiar la recaudación tributaria, puede tener efectos perjudiciales sobre la inversión privada. Además si hay que elegir un aumento de impuestos, en este artículo mostramos evidencia a favor de que incrementos del impuesto sobre el consumo son menos perjudiciales para la inversión privada que aumentos en el impuesto sobre la renta.

Los resultados mostraron efectos negativos entre la acción gubernamental y la política tributaria. No obstante, la evidencia a favor de la conexión de estas variables con el crecimiento económico es abundante. Aquí suponemos que no es la cantidad de gasto, sino la calidad de este, la clave determinante del desarrollo; entendido este último como la capacidad para promover el bienestar social. Lo mismo puede ocurrir con la política tributaria, los impuestos altos desalientan la demanda de inversión o de consumo, como en el caso de los impuestos regresivos y con lo cual se puede afectar de manera negativa el gasto social.

\section{REFERENCIAS BIBLIOGRÁFICAS}

Arévalo, Guillermo. (2014). Ecuador: economía y política de la revolución ciudadana, evaluación preliminar. Apuntes del CENES. 109-134.

Brito, L. e Iglesias, E. (2017a). Inversión privada, gasto público y presión tributaria en América Latina. Congreso anual 2016 de la Asociación Peruana de Economía (APE). Lima, Perú. Pontificia Universidad Católica del Perú.

Brito, L. e Iglesias, E. (2017b). Inversión privada, gasto público y presión tributaria en Europa. Conferencia Científica Anual - UNICA 2016 de la Universidad de Ciego de Ávila. Cuba.

Caballero, E. y López, J. (2012). Impuesto sobre la Renta e inversión privada en México. Investigación Económica. LXXI.

Carrasco, Carlos. (2012). Una nueva política fiscal para el buen vivir. La equi- 
dad como soporte del pacto fiscal. Servicio de Rentas Internas. Ecuador. CEPAL. (2014). Panorama fiscal de América Latina y el Caribe. CEPAL. Naciones Unidas. Chile.

Gutiérrez, H. (1996). Estimación del factor de crowding out entre inversión pública y privada para Chile. Estudios de Economía. 23(1), 1-29

Harberger, A. C. (1964). The measurement of Waste. American Economic Review LIV, 58-76.

Hausman, J.A. (1978). Specification test in econometrics. Econometrica, 46. 1251- 1271.

Ibarra, C. (2008). Mexico's slow growth paradox. Cepal Review, 95

Ibarra, C. (2009). Capital flows, preemptive policy, and the external constraint in Mexico. Working Paper, Departamento de Economía, Universidad de las Américas. México.

Jiménez, Juan. (2015). Desigualdad, concentración del ingreso y tributación sobre las altas rentas en América Latina. Centro de Estudios Fiscales. CEPAL, 134.

López, J., Spanos, A. y Sánchez, A. (2011). Macroeconomic linkages Mexico. Metroeconomica. 62(2), 356-385.

Mendoza, E., Milesi-Ferretti, G., Asea, P. (1997). On the ineffectiveness of tax policy in altering long-run growtl: Harberger's superneutrality conjecture. Journal of Public Economics. 66(1), 99-126.

Pecho-Trigueros, M. y Peragón-Lorenzo, L. A. (2013), Estimación de la carga tributaria efectiva sobre la inversión en América Latina. Centro Interamericano de Administraciones Tributarias. CIAT. Panamá.

Pecorino, P. (1993). Tax structure adn growth in a model with human capital. Journal of Public Economics. 52, 251-271.

Pérez Rodríguez, O. (2011). Modelo kaleckiano de crecimiento con gasto público. Inversión en educación y salud como propulsores del desarrollo. Revista CIFE, 13(19), 13-32.

Requeijo, J. (1995). Economía Mundial: un análisis entre dos siglos. Madrid: Editorial McGrawHill.

Wooldridge, J. (2010). Introducción a la econometría. Un enfoque moderno. México. 